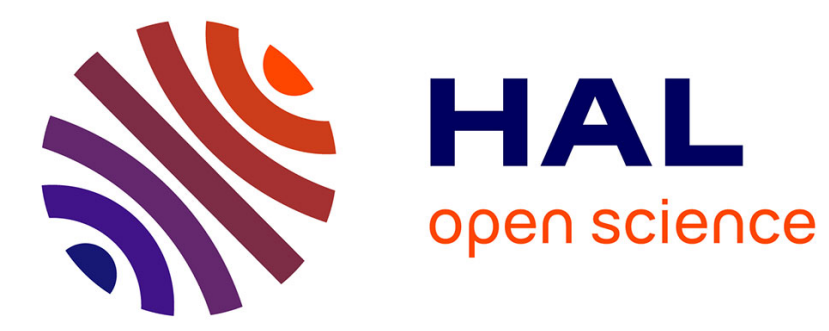

\title{
La perception de jeunes lesbiennes et gais concernant l'attitude de leurs parents à l'égard de leur homosexualité
}

\author{
Elodie Charbonnier, Pierluigi Graziani
}

\section{- To cite this version:}

Elodie Charbonnier, Pierluigi Graziani. La perception de jeunes lesbiennes et gais concernant l'attitude de leurs parents à l'égard de leur homosexualité. Canadian Journal of Community Mental Health, 2011. hal-01682922

\section{HAL Id: hal-01682922 \\ https://hal.science/hal-01682922}

Submitted on 12 Jan 2018

HAL is a multi-disciplinary open access archive for the deposit and dissemination of scientific research documents, whether they are published or not. The documents may come from teaching and research institutions in France or abroad, or from public or private research centers.
L'archive ouverte pluridisciplinaire HAL, est destinée au dépôt et à la diffusion de documents scientifiques de niveau recherche, publiés ou non, émanant des établissements d'enseignement et de recherche français ou étrangers, des laboratoires publics ou privés. 


\title{
La perception de jeunes lesbiennes et gais concernant l'attitude de leurs parents à l'égard de leur homosexualité
}

\author{
Élodie Charbonnier \\ Université de Nimes/Aix-Marseille Université \\ Pierluigi Graziani \\ Université de Nimes/Aix-Marseille Université
}

\begin{abstract}
RÉSUMÉ
Cette étude vise à mieux appréhender les facteurs de vulnérabilité des jeunes lesbiennes et gais en lien avec les attitudes de leurs parents vis-à-vis de leur homosexualité. Elle a été menée en France auprès de 82 jeunes lesbiennes et gais. Au sein du groupe, $66 \%$ affirment avoir déjà eu des idées suicidaires tandis que $31 \%$ disent avoir déjà fait au moins une tentative de suicide. Ces résultats mettent en lumière la vulnérabilité de cette population. Par ailleurs, les résultats montrent qu'une attitude paternelle perçue comme rejetante est corrélée à la présence d'idées suicidaires et à la réalisation d'au moins une tentative de suicide. De plus, lorsque la réaction maternelle est perçue négativement, elle est corrélée à des difficultés d'interaction sociale et à un fort niveau d'inquiétude. L'attitude parentale perçue par les jeunes lesbiennes et gais constitue donc un élément probant de la détresse et du risque suicidaire élevé dans ce groupe.
\end{abstract}

Mots clés : homosexualité, coming out, attitude parentale, jeunes, tentative de suicide, idéation suicidaire

[

\section{Suicide et homosexualité}

Les premières données rendant compte de la relation homosexualité-suicide émanent du Report of the Secretary's Task Force on Youth Suicide du département étatsunien de la santé et des services humains. Ces données suggèrent que les jeunes gais font au moins deux à trois fois plus de tentatives de suicide que

Élodie Charbonnier, psychologue clinicienne, doctorante en psychologie clinique et psychopathologie, Unimes/Aix-Marseille Univ, LPS EA 849, 30021, Nimes, France ; Pierluigi Graziani, professeur de psychologie clinique et psychopathologie, Unimes/ Aix-Marseille Univ, LPS EA 849, 30021, Nimes, France.

Cette étude bénéficie du soutien du Groupement d'Étude et de Prévention du Suicide (GEPS), et d'une subvention de la Fondation Pfizer, pour la santé de l'enfant et de l'adolescent.

La correspondance concernant cet article devrait être acheminée à Élodie Charbonnier. Courriel : charbonnier.elodie@gmail.com 
les hétérosexuels, et qu'ils représenteraient jusqu'à $30 \%$ des suicides des adolescents (Gibson, 1989). Ce rapport précise que les problèmes familiaux seraient un des facteurs permettant de mieux comprendre leur risque suicidaire élevé. Par la suite, diverses recherches ont confirmé ces résultats pour les jeunes gais mais aussi pour les lesbiennes (D’Augelli, Hershberger et Pilkington, 1998 ; Garofalo, Wolf, Kessel, Palfrey et DuRant, 1998 ; Garofalo, Wolf, Wissow, Woods et Goodman, 1999 ; Russell et Joyner, 2001).

Selon une récente étude étatsunienne, 17,2\% des lesbiennes, gais, bisexuelles ou bisexuels (18-26 ans) ont déclaré avoir eu des idées suicidaires et 4,9\% ont déclaré avoir tenté de se suicider durant les 12 derniers mois, contre respectivement $6,3 \%$ et $1,6 \%$ des hétérosexuelles et hétérosexuels du même âge (Silenzio, Peña, Duberstein, Cerel et Knox, 2007). Le rapport du Suicide Prevention Resource Center (2008) valide ces données et souligne que les jeunes gais et lesbiennes ont entre 1,5 et trois fois plus de risque d'avoir des idées suicidaires, et sont jusqu'à sept fois plus susceptibles de tenter de se suicider que leurs pairs hétérosexuels. Des enquêtes et études canadiennes confirment que les taux d'idéations suicidaires et de tentatives de suicide sont plus marqués chez les adultes lesbiennes, gais, bisexuelles et bisexuels que chez les hétérosexuelles et hétérosexuels (Bagley et Tremblay, 1997 ; Clermont et Lacouture, 2000 ; Otis et al., 2002)

Malgré ces données alarmantes en provenance des États-Unis et du Canada, les recherches en France s'intéressant à cette question sont peu nombreuses. Selon une des premières enquêtes française menée par voie de presse en 2001, 17 \% des gais déclarent avoir déjà fait une tentative de suicide au cours de leur vie, ce taux étant d'autant plus marqué chez les plus jeunes (27\% pour les moins de 20 ans) (Adam, 2001). La part des hommes qui ont réalisé une tentative de suicide a augmenté entre 2000 et 2004, atteignant un taux de $19 \%$ pour la totalité de l'échantillon et de $32 \%$ pour les moins de 20 ans (Velter, 2007). Cette sursuicidalité a été confirmée par une récente étude menée par Bourdet-Loubère et Pugnière (2011) dans laquelle les jeunes se déclarant homosexuels étaient 12 fois plus nombreux que les autres à réaliser une tentative de suicide.

Ce n'est qu'en 2008 que le ministère du Travail, de l'Emploi et de la Santé a mis l'accent sur la nécessité de « lutter contre la souffrance psychique liée à l'homosexualité » et d'accentuer le « repérage et la prévention de la crise suicidaire et de la souffrance psychique chez les jeunes, plus particulièrement chez les jeunes homosexuels » (Ministère français du Travail, de l'Emploi et de la Santé, 2008). Ce projet met en lumière les résultats d'une enquête française menée auprès de 16883 participantes et participants (15-75 ans) dans le cadre du « Baromètre Santé 2005 », qui démontre une prévalence d'épisodes dépressifs caractérisés au cours des 12 derniers mois plus importante chez les gais et bisexuels que chez les hétérosexuels (10,4\% vs 3,9\%). On voit également une disparité en ce qui concerne le taux de tentatives de suicide au cours de la vie $(7,5 \%$ vs $2,5 \%)$. Par contre, les lesbiennes et les bisexuelles présentent des résultats similaires aux hétérosexuelles en termes d'épisodes dépressifs, de pensées suicidaires et de tentatives de suicide (Jouvin, Beaulieu-Prévost et Julien, 2007).

Ces dernières données sont en contradiction avec celles de Lhomond et Saurel-Cubizolles (2003) qui attestent que $25 \%$ des femmes (25-59 ans) qui ont eu des rapports avec d'autres femmes ont fait une tentative de suicide au cours de leur vie. La prévalence des conduites suicidaires est de $14 \%$ pour les femmes qui déclarent être attirées par les femmes sans avoir eu de rapport homosexuel et de $6 \%$ pour les femmes exclusivement hétérosexuelles. Ces résultats divergents peuvent être expliqués par des différences méthodologiques. La première enquête (Jouvin et al., 2007), se basant sur les comportements sexuels au cours des 12 derniers mois, exclut les personnes inactives sexuellement dans l'année, qui par contre sont incluses 
dans la seconde (Lhomond et Saurel-Cubizolles, 2003) qui se centre sur les comportements ou les attirances au cours de la vie.

Les lesbiennes et les gais présentent plus de facteurs de risque que les hétérosexuelles et hétérosexuels. Ainsi, elles et ils présentent fréquemment un manque de soutien, des difficultés scolaires, des problèmes familiaux, des conduites addictives et des troubles psychiatriques (Kitts, 2005), mais également une peur du harcèlement, des mauvais traitements (Huebner, Rebchook et Kegeles, 2004), de la stigmatisation et de la discrimination (Garofalo et Katz, 2001). Les lesbiennes et les gais font l'objet de plus de harcèlement sexuel (Williams, Connolly, Pepler et Craig, 2005), d'abus sexuels et de violence physique que les hétérosexuelles et hétérosexuels (Corliss, Cochran et Mays, 2002 ; Saewyc, Bearinger, Blum et Resnick, 1999 ; Saewyc et al., 2006 ). Selon Mays et Cochran (2001), plus des trois quarts des lesbiennes, gais, bisexuelles et bisexuels déclarent avoir vécu des discriminations, essentiellement en lien avec leur orientation sexuelle. Celles-ci sont omniprésentes dans le milieu scolaire et prennent essentiellement la forme de propos homophobes (Chamberland, Émond, Julien, Otis et Ryan, 2010). Ces expériences de discriminations favoriseraient une plus grande vulnérabilité à la dépression, à l'anxiété et aux troubles psychiatriques (Kessler, Mickelson et Williams, 1999 ; Cochran, 2001). Les lesbiennes, gais, bisexuelles et bisexuels ayant subi une agression physique ou sexuelle au cours de leur vie auraient plus de risque de faire une tentative de suicide (DuRant, Krowchuk et Sinal, 1998 ; Saewyc et al., 2006 ; Warner et al., 2004)

Les facteurs de protection sont aussi indispensables à prendre en considération pour mieux appréhender leur risque suicidaire. Un jeune qui présente des facteurs de protection (tels que le soutien de sa famille et de l'école) a une plus faible probabilité de réaliser une tentative de suicide (Saewyc et al., 2007). Ces facteurs ont un effet protecteur, ils modèrent la relation entre les facteurs de risques et les conduites suicidaires. Or, les jeunes lesbiennes, gais, bisexuelles et bisexuels présentent moins de facteurs de protection que les jeunes hétérosexuelles et hétérosexuels (Saewyc et al., 2009) : elles et ils possèdent de moins bon liens familiaux, reçoivent moins d'attention de la part de leurs professeurs et professeures ou des autres adultes, et se sentent moins en sécurité à l'école que les hétérosexuelles et hétérosexuels (Eisenberg et Resnick, 2006).

\section{Le coming out}

Le coming out (l'annonce de son homosexualité) est une situation de stress spécifique aux lesbiennes et aux gais. Cette annonce est souvent difficile et stressante (Iwasaki et Ristock, 2007 ; Savin-Williams, 2001 ; Willoughby, Malik et Lindahl, 2006), mais faire son coming out est une étape nécessaire pour que les jeunes non hétérosexuels structurent leur identité en se définissant comme gai, lesbienne, bisexuel ou bisexuelle lors de cette révélation. L'identité homosexuelle (ou bisexuelle) est mouvante, se construisant au fil des interactions sociales. Chaque coming out va être l'occasion d'une construction ou d'une reconstruction de cette identité (Mellini, 2009). Personne n'étant uniquement homosexuel, chaque individu dispose de plusieurs identités (Eribon, 1999), qui elles aussi se construiront au fil des interactions (Camilleri, 1990). Qui plus est, en analysant la littérature, Savin-Williams (1998) en conclut que les personnes n'ayant pas encore dévoilé leur homosexualité se situeraient dans une position inconfortable, dans un état d'anxiété, dominées par la peur que leurs parents découvrent leur orientation sexuelle, et ceci jusqu'à ce qu'elles sortent du secret.

Le coming out nécessite de s'exposer au regard de l'autre et à un éventuel rejet. L'idée de dévoiler son homosexualité à ses parents suscite de la peur et de l'anxiété (Savin-Williams et Esterberg, 2000). Des 
conséquences négatives sont souvent anticipées, reculant cette annonce. Selon Savin-Williams (2001), un jeune (17-25 ans) sur trois a annoncé son homosexualité à son père, et deux jeunes sur trois ont fait leur coming out auprès de leur mère. La même tendance est observée en France, parmi les 16-20 ans, 61 \% déclarent ne pas avoir dévoilé leur homosexualité à leur père, $49 \%$ ne pas l'avoir fait auprès de leur mère (Schiltz, 1995). Différentes stratégies identitaires peuvent être employées pour affirmer ou cacher son orientation sexuelle telles que le déni, la clandestinité, l'arrangement ou encore l'affichage (Mellini, 2009).

Ceux et celles qui ont effectué leur coming out auraient quatre fois plus de risque de faire une tentative de suicide au cours de leur vie que les jeunes qui n'ont pas divulgué leur homosexualité (D'Augelli et al., 1998). Plus l'âge de l'annonce est précoce, plus le risque suicidaire est élevé (Remafedi, Farrow et Deisher, 1991), les plus jeunes étant moins aptes à faire face à l'isolement et à la stigmatisation pouvant faire suite à leur coming out.

\section{Les attitudes parentales}

La perception que les enfants et les adolescents et adolescentes ont d'eux-mêmes est en partie basée sur la manière dont leurs parents les perçoivent. L'acceptation ou le rejet des parents peut influencer la représentation qu'ils et elles ont d'eux-mêmes ou affecter leur santé (Rohner, 2004). Un faible soutien familial a été mis en lien avec l'installation de signes dépressifs et le recours à des substances (Aseltine, Gore et Colten, 1998).

Les réactions familiales suite au coming out sont variées. Pour $68 \%$ des gais et $55 \%$ des lesbiennes, leur mère a réagi négativement à leur coming out. Des résultats similaires sont observés pour la réaction du père (Willoughby et al., 2006). Selon Pilkington et D'Augelli (1995), environ $30 \%$ des lesbiennes, gais, bisexuelles et bisexuels (15-21 ans) ont rencontré de la violence verbale de la part de leur famille par rapport à leur orientation sexuelle et $10 \%$ ont été agressés physiquement. Dans l'enquête française menée par SOS Homophobie (2004), $67 \%$ des répondantes et répondants ont déclaré avoir rencontré des épisodes de rejet de la part de leur famille lors de leur coming out. Pour $33 \%$, ces rejets provenaient de leur mère, pour $29 \%$ de leur père et pour $19 \%$ de leur fratrie.

Des attitudes parentales de rejet peuvent être impliquées dans le développement de troubles relationnels, de troubles de l'attachement, de difficultés scolaires, d'abus de substances ou de dépression (Hale, Van Der Valk, Engles et Meeus, 2005 ; Pedersen, 1994 ; Piko, 2000). A contrario, des attitudes parentales positives vis-à-vis de leur homosexualité sont mises en lien chez les jeunes lesbiennes, gais, bisexuelles et bisexuels avec le développement de comportements sociaux harmonieux (Rohner, Khaleque et Cournoyer, 2003) et de leur bien-être (Floyd, Stein, Harter, Allison et Nye, 1999). Les attitudes parentales positives sont corrélées négativement avec le risque de réaliser une tentative de suicide (Eisenberg et Resnick, 2006) ou de développer de l'homophobie intériorisée (Nungesser, 1983 ; Savin-Williams, 1989) ou des comportements sexuels à risque (Vincke, Bolton, Mak et Blank, 1993).

Cette étude se propose d'étudier les facteurs de vulnérabilité des jeunes lesbiennes et gais en mettant l'accent sur les liens existants entre la manière dont les jeunes lesbiennes et gais perçoivent l'attitude de leurs parents vis-à-vis de leur homosexualité et diverses variables. Nous interrogerons plus particulièrement les corrélations existantes entre l'attitude parentale perçue et la présence d'idéations suicidaires, de tentative de suicide, d'inquiétudes et de difficultés relationnelles. Notre hypothèse est la suivante : des réactions 
parentales perçues comme positives seraient liées négativement à ces variables, alors qu'à l'inverse, elles entretiendraient des liens positifs avec des attitudes parentales perçues négativement.

\section{MÉTHODOLOGIE}

\section{Répondantes et répondants}

Cette étude a été menée en France auprès de 82 participantes et participants s'identifiant comme lesbienne ou gai (33 femmes et 49 hommes) qui ont révélé leur homosexualité à leurs parents. Nous nous sommes appuyés sur trois mesures de l'orientation sexuelle (Dean et al., 2000) comme critère de sélection. Pour participer à cette étude, les individus devaient avoir eu au moins une expérience homosexuelle (comportement), une relation homosexuelle (sentiments) et se définir comme étant lesbienne ou gai (vécu subjectif). L'âge des répondantes et répondants est compris entre 18 et 25 ans ( $m=21,2$ ans ; $E T=2,3)$.

Les personnes interrogées ont été rencontrées via internet dans différents sites de rencontre homosexuels. Des messages privés ont été envoyés à l'ensemble des profils précisant la thématique de l'étude (à savoir les attitudes parentales lors de l'annonce de son homosexualité), le cadre de la recherche (étude menée lors d'une recherche de Master 2 en psychologie), ainsi que la procédure à suivre. Les personnes intéressées ont répondu anonymement à un questionnaire, après avoir signé un formulaire de consentement. Les analyses effectuées, un rapport leur a été transmis énonçant les principaux résultats de l'étude.

\section{Questionnaires utilisés}

A l'aide de questionnaires explicitées dans les paragraphes suivantes, cinq variables ont été mesurées : l'inquiétude ressentie dans diverses situations, la réalisation d'au moins une tentative de suicide, la présence d'idées suicidaires, leurs interactions avec les autres et la perception qu'ils ont de l'attitude de leurs parents à l'égard de leur homosexualité. L'ensemble des analyses statistiques ont été réalisées à l'aide du logiciel SPSS 16.

Questionnaire des domaines d'inquiétude (Worry Domains Questionnaire). La version anglaise (Tallis, Eysenck et Mathews, 1992) de ce questionnaire, validée auprès d'étudiants (Davey, 1993), est composée de 25 items correspondant à cinq domaines d'inquiétude. La version française traduite et validée auprès d'étudiants (Dugas, Letarte, Rhéaume, Freeston et Ladouceur, 1995) et de répondantes et répondants cliniques et non cliniques (Ladouceur et al., 1999) a été utilisée dans cette recherche. La version française a une bonne consistance interne de 0,9 et une fidélité test-retest à quatres semaines de 0,6 (Dugas et al., 1995). Elle comprend 30 items, cotés sur une échelle Likert allant de zéro (pas du tout) à quatre (extrêmement), et explore six domaines d'inquiétude : « relation avec les autres », « manque de confiance », « futur sans objectif », « incompétence au travail », « menace financière » et « menace physique ». Dans l'analyse des résultats, les moyennes obtenues par Ladouceur et al. (1999) ont servi de score de comparaison.

Questionnaire des attitudes parentales perçues (en annexe). Ce questionnaire de dix items (cinq concernant l'attitude du père et les cinq autres celle de la mère) a été construit afin d'évaluer la manière dont les jeunes lesbiennes et gais ont perçu l'attitude de leurs parents à l'égard de leur homosexualité. Il a été constitué à partir des thématiques redondantes abordées par les jeunes lesbiennes et gais au cours de deux 
groupes de discussion réalisés au sein d'une association à Marseille (France) dont le thème était « le coming out auprès des parents ». Dans ce questionnaire, les participantes et participants ont répondu aux items sur une échelle Likert allant de 1 (très mauvais) à 5 (très bon). Ils ont évalué la réaction de leurs parents lors de l'annonce de leur homosexualité, leur attitude actuelle à l'égard de leur orientation sexuelle, ou encore la qualité des conversations qu'ils et elles avaient avec eux lorsqu'ils et elles évoquaient leur homosexualité.

L'analyse factorielle des items de ce questionnaire en moindres carrés non pondérés avec une rotation oblique a fait ressortir trois facteurs. Le dernier ayant une importance faible, seuls deux facteurs ont été retenus. Ils expliquent $63,8 \%$ de la variance totale (variance expliquée du facteur $1=40,9 \%$, variance expliquée du facteur $2=22,9 \%)$, et sont fortement corrélés $(r=0,3 ; p \leq 0,01)$. Le premier facteur est saturé par cinq items (item 6,7,8, 9 et 10) qui correspondent aux questions portant sur la mère; il a été intitulé « attitude maternelle ». Le second est également saturé par cinq items (item 1, 2, 3, 4 et 5), qui portent sur le père ; il a été nommé « attitude paternelle ». La consistance interne du questionnaire est satisfaisante, l'alpha de Cronbach pour les 10 items étant de 0,8 . L'homogénéité des deux facteurs est également satisfaisante ( $\alpha$ de Cronbach du facteur $1=0,9 ; \alpha$ de Cronbach du facteur $2=0,8$ ).

Questionnaire des conduites interpersonnelles (Scale for Interpersonal Behaviour). La version hollandaise initiale (Arrindell, De Groot et Walburg, 1984) a fait l'objet de diverses validations auprès de populations contrôles (Arrindell et Van der Ende, 1985 ; Arrindell et al., 1999) et de populations cliniques (Arrindell et Van der Ende, 1985 ; Arrindell, Sanderman, Van der Molen, Van der Ende et Mersch, 1988). Traduite et adaptée en français, elle a été validée par Bouvard et al. (1999) à partir de 166 participants et participantes ayant des troubles de l'assertivité, et 150 individus contrôles comparables pour l'âge, le sexe et le niveau d'étude. Les moyennes obtenues par les individus contrôles ont été comparées à celles de nos participantes et participants. La consistance interne des deux sous-échelles a été étudiée sur des populations contrôles et des sujets anxieux sur les différentes versions. Elle varie de 0,8 à 1 pour l'échelle de gêne et de 0,7 à 0,9 pour l'échelle de performance. La fidélité test-retest ( 20 à 40 jours) est meilleure pour l'échelle de gêne mais les valeurs restent satisfaisantes quelle que soit la version.

Ce questionnaire de 50 items permet d'appréhender deux dimensions : la performance (la probabilité de la personne à s'engager dans une réponse assertive) et la gêne (le degré de gêne lorsque l'individu réalise la situation décrite). Pour chaque item, les participantes et participants doivent répondre à ces deux dimensions. Par exemple, à l'item 1, « Commencer une conversation avec un-e étranger-ère », elles et ils doivent répondre à deux questions :

1. «Etes-vous tendu-e ?»: $1=$ pas du tout, $2=$ un peu, $3=$ moyennement, $4=$ beaucoup, $5=$ énormément.

2. «Le faites-vous ?»: $1=$ jamais, $2=$ rarement, $3=$ parfois, $4=$ d'habitude, $5=$ toujours.

Plus le score de gêne est élevé, plus la répondante ou le répondant est considéré-e en difficulté face à la situation mentionnée. À l'inverse, plus le score de performance est haut, moins il ou elle sera amené-e à éviter la situation. Les deux échelles sont reparties en quatre sous échelles : SE1 : « exposer des sentiments négatifs et défendre ses droits »; SE2 : « expression d'une limitation personnelle »; SE3 :« initiative de l'assertivité »; SE4 :« donner et recevoir des compliments ».

Présence d'idéations et d'au moins une tentative de suicide (ou plus). La présence d'idées suicidaires et de tentative de suicide a été évalué à l'aide de deux items dichotomiques : « Avez-vous déjà tenté de mettre 
fin à vos jours au cours de votre vie ? » et « Avez-vous déjà pensé à mettre fin à vos jours au cours de votre vie ? ». Pour chaque item, deux modalités de réponses ont été proposées aux participantes et participants : «Oui » et « Non », cotées respectivement 1 et 0 .

\section{RÉSULTATS}

\section{Niveau d'inquiétude}

Les répondantes et les répondants ont obtenu un score moyen de 61,2 (ÉT $=22,8)$ au Questionnaire des domaines d'inquiétude. Il n'y a pas de différence statistiquement significative entre le score d'inquiétude des gais et des lesbiennes $\left(F[81]_{\text {score inquiétude }}=2,4 ; p=\mathrm{NS}\right)$ et ceci pour toutes les sous-échelles $\left(\mathrm{F}[81]_{\text {relation aux }}\right.$ autres $=0,5 ; p=\mathrm{NS} / F[81]_{\text {manque de confiance }}=1,1 ; p=\mathrm{NS} / F[81]_{\text {futur sans objectif }}=2,6 ; p=\mathrm{NS} / F[81]_{\text {incompétence au }}$ travail $\left.=2,7 ; p=\mathrm{NS} / F[81]_{\text {menace financière }}=1,9 ; p=\mathrm{NS} / F[81]_{\text {menace physique }}=0,5 ; \mathrm{p}=\mathrm{NS}\right)$.

Ladouceur et al. (1999) ont mené une étude auprès de trois groupes d'individus : des personnes atteintes de trouble anxieux généralisé (TAG) $(n=24)$, des personnes atteintes d'autres troubles anxieux $(n=38)$ et des personnes contrôles $(n=20)$. Les moyennes obtenues par ces répondantes et répondants au Questionnaire des domaines d'inquiétude ont été comparées à celles de nos participantes et participants à l'aide d'un test de Student sur échantillon unique (en prenant comme valeur de comparaison les moyennes des auteurs). La moyenne des jeunes gais et lesbiennes de notre échantillon au Questionnaire des domaines d'inquiétude est statistiquement équivalente à celle obtenue par les auteurs pour des patients et patientes présentant un TAG (cf. tableau 1) et supérieure au groupe contrôle. Les participantes et participants de notre recherche apparaissent donc avoir un niveau d'inquiétude élevé, proche de celui des individus présentant un trouble anxieux généralisé.

\section{Tableau 1}

Moyennes, écarts types et comparaisons ( $t$-Student à échantillon unique) entre les scores des lesbiennes et des gais au Questionnaire des domaines d'inquiétude et trois groupes d'individus*

\begin{tabular}{cccc}
\hline $\begin{array}{c}\text { Lesbiennes et gais } \\
(n=82)\end{array}$ & $\begin{array}{c}\text { TAG }(n=24) \\
\text { (Ladouceur } \text { et al. }[1999])\end{array}$ & $\begin{array}{c}\text { Autre troubles anxieux }(n=38) \\
\text { (Ladouceur } \text { et al. }[1999])\end{array}$ & $\begin{array}{c}\text { Contrôle }(n=20) \\
\text { (Ladouceur } \text { et al. }[1999])\end{array}$ \\
\hline $\mathbf{6 1 , 2} \mathbf{( 2 2 , 8 )}$ & $\mathbf{5 6 , 9}(\mathbf{2 2})$ & $44,3(21,8)$ & $21,1(16,1)$ \\
& $t(81)=1,7$ & $t(81)=6,7$ & $t(81)=15,9$ \\
& $p=\mathrm{NS}$ & $\boldsymbol{p} \leq \mathbf{0 , 0 0 1}$ & $\boldsymbol{p} \leq \mathbf{0 , 0 0 1}$ \\
\hline
\end{tabular}

*personnes souffrant de TAG, d'autres troubles anxieux et groupe contrôle (les moyennes de ces trois groupes sont issues de l'étude de Ladouceur et al. [1999])

\section{Idées suicidaires et tentatives de suicide}

Une proportion de $66 \%$ des participantes et participants affirment avoir déjà eu des idées suicidaires, et $31 \%$ déclarent avoir déjà tenté de mettre fin à leurs jours. Les résultats des gais et des lesbiennes sont statistiquement équivalents en ce qui concerne la présence de tentatives de suicide $(t[80]=0,03 ; p=$ NS) et d'idéations suicidaires $(t[80]=1,1 ; p=\mathrm{NS})$. 


\section{Attitudes parentales perçues}

Les notes allant pour chaque item de 1 (très mauvaise réaction) à 5 (très bonne réaction), la moyenne globale au questionnaire d'attitude parentale perçue des répondantes et répondants est de 2,7 (ÉT=1,1) ce qui correspond à une attitude se situant entre mauvaise et moyenne. La moyenne de l'attitude paternelle perçue (facteur 2) est de 2,2 (ÉT = 1,3). Elle est donc en moyenne jugée mauvaise, alors que l'attitude maternelle (facteur 1) est considérée comme moyenne $(m=3,1 ; E ́=1,5)$ par les participants et participantes. Au vu des écarts types élevés, des attitudes parentales très variées peuvent être perçues par les lesbiennes et les gais de notre échantillon. Le score du facteur 2 est statistiquement inférieur à celui du facteur $1(t[80]=4,7$; $p<0,0001)$. Les mères seraient donc perçues comme étant plus acceptantes que les pères. Il n'y a pas de différences statistiquement significatives entre les hommes et femmes que ce soit pour le facteur $2(t[80]=$ $1,7 ; p=\mathrm{NS})$ ou pour le facteur $1(t[80]=1,2 ; p=\mathrm{NS})$.

Les répondants et répondantes ont évalué la réaction de leurs parents lors de leur coming out et leur attitude actuelle. Lors du coming out, la perception de l'attitude parentale est statistiquement équivalente pour les deux parents (cf. tableau 2). Par contre, la perception que les jeunes ont de leur réaction actuelle est significativement différente. Celle de la mère est jugée plus positive que celle du père. Ainsi, même si, lors de l'annonce, l'attitude des deux parents est perçue de la même manière, celle de la mère est vécue comme plus positive que celle du père avec le temps. Toutefois, les deux évoluent positivement.

\section{Tableau 2}

Moyenne, écart type et comparaison ( $t$-Student) entre les scores des pères et des mères à quatre items du Questionnaire d'attitudes parentales perçues $(n=82)$

\begin{tabular}{lcc}
\hline \multicolumn{3}{c}{ Attitude parentale perçue lors du coming out } \\
\hline Attitude perçue du père (item 1) & $m=3,2, E T=1,3$ & $t(80)=1,3$ \\
Attitude perçue de la mère (item 6) & $m=2,9, E T=1,4$ & $p=\mathrm{NS}$ \\
\hline & Attitude parentale perçue actuellement & \\
\hline Attitude perçue du père (item 2) & $m=3,6, E ́ E=1,2$ & $\boldsymbol{t}(\mathbf{8 0})=\mathbf{- 2}$ \\
Attitude perçue de la mère (item 7) & $m=3,9, E T=1,2$ & $\boldsymbol{p} \leq \mathbf{0 , 0 5}$ \\
\hline
\end{tabular}

Le coming out auprès de la mère a été effectué en moyenne à 17,7 ans ( $E T=2,2$ ans) et en moyenne à 18,1 ans auprès du père ( $E$ T $=2,3$ ans). L'âge que les jeunes avaient lors de la révélation à leur père ou à leur mère est statistiquement différent $(t[80]=-2,6 ; p<0,01)$. Le coming out semble être effectué dans un premier temps à la mère puis au père.

\section{Score de gêne en interaction et de performance sociale}

Les moyennes de nos participantes et participants ont été comparées à celles obtenues par Bouvard et al. (1999) lors de la validation française du Questionnaire de conduites interpersonnelles auprès de 150 individus contrôles issus de la population générale à l'aide d'un test de Student sur échantillon unique prenant pour valeur de référence les moyennes obtenues par les auteurs. La moyenne des jeunes gais et lesbiennes de 
notre échantillon à l'échelle de gêne est statistiquement différente de celle du groupe contrôle (cf. tableau 3). Nos participantes et participants présentent un score de gêne dans leurs interactions sociales plus marqué que celui obtenu par les auteurs. Elles et ils sont particulièrement gênés dans les situations au cours desquelles elles et ils doivent s'exposer à des sentiments négatifs et défendre leurs droits (SE1), être assertifs (SE3) ou encore donner ou recevoir des compliments (SE4). Mais les participantes et les participants sont à même d'exprimer une limitation personnelle (SE2) sans ressentir une gêne marquée.

La moyenne à l'échelle de performance des répondantes et répondants est statistiquement équivalente à celle des individus du groupe contrôle (cf. tableau 3). Malgré la gêne que ces jeunes ressentent dans certaines situations, les gais et les lesbiennes de cette recherche ne semblent pas avoir de difficultés à faire ou accepter un compliment, à exprimer leurs difficultés ou encore leurs opinions.

Tableau 3

Moyennes, écarts types et comparaisons ( $t$-Student à échantillon unique) entre les moyennes des lesbiennes et des gais au Questionnaire des conduites interpersonnelles et celles d'individus contrôles*

\begin{tabular}{|c|c|c|c|}
\hline & $\begin{array}{c}\text { Gais et lesbiennes } \\
\text { de notre } \\
\text { échantillon }(n=82) \\
\text { Moyenne (écart type) }\end{array}$ & $\begin{array}{l}\text { Individus contrôles } \\
\text { Bouvard et al. }(1999) \\
\quad(n=150) \\
\text { Moyenne (écart type) }\end{array}$ & $t$ \\
\hline Gêne en interaction & $117,8(35,1)$ & $104,1(31,8)$ & $\begin{array}{c}t(81)=3,5 \\
p \leq 0,001\end{array}$ \\
\hline $\begin{array}{l}\text { Exposer des sentiments négatifs ou } \\
\text { défendre leurs droits (SE1) }\end{array}$ & $38,2(12,2)$ & $34,8(11,1)$ & $\begin{array}{l}t(81)=2,5 \\
p \leq 0,05\end{array}$ \\
\hline $\begin{array}{l}\text { Exprimer une limitation personnelle } \\
\text { (SE2) }\end{array}$ & $29,6(10,2)$ & $29,6(8,9)$ & $\begin{array}{c}t(81)=-0,004 \\
p=\mathrm{NS}\end{array}$ \\
\hline Doivent être assertifs (SE3) & $21,6(7,5)$ & $18,9(6,8)$ & $\begin{array}{c}t(81)=3,3 \\
p \leq 0,001\end{array}$ \\
\hline $\begin{array}{l}\text { Donner ou recevoir des compliments } \\
\text { (SE4) }\end{array}$ & $19,6(6,3)$ & $16,5(6,2)$ & $\begin{array}{c}t(81)=4,5 \\
p \leq 0,001\end{array}$ \\
\hline Performance sociale & $155,2(29,7)$ & $155,2(22,8)$ & $\begin{array}{c}t(81)=0 \\
p=\mathrm{NS}\end{array}$ \\
\hline $\begin{array}{l}\text { Exposer des sentiments négatifs ou } \\
\text { défendre leurs droits (SE1) }\end{array}$ & $42,2(10)$ & $42,1(8)$ & $\begin{array}{c}t(81)=0,1 \\
p=\mathrm{NS}\end{array}$ \\
\hline $\begin{array}{l}\text { Exprimer une limitation personnelle } \\
\text { (SE2) }\end{array}$ & $48,1(9,4)$ & $49,4(7,3)$ & $\begin{array}{l}t(81)=-1,2 \\
p=\mathrm{NS}\end{array}$ \\
\hline Doivent être assertifs (SE3) & $28(6,4)$ & $28,6(5,5)$ & $\begin{array}{c}t(81)=-0,8 \\
p=\mathrm{NS}\end{array}$ \\
\hline $\begin{array}{l}\text { Donner ou recevoir des compliments } \\
\text { (SE4) }\end{array}$ & $24,4(6)$ & $23,2(4,7)$ & $\begin{array}{l}t(81)=1,8 \\
\quad p=\mathrm{NS}\end{array}$ \\
\hline
\end{tabular}

*obtenues par Bouvard et al. (1999) lors de la validation française du questionnaire 


\section{Relations entre le score au Questionnaire d'attitudes parentales perçues et les différentes variables}

La perception de l'attitude paternelle (facteur 2) est corrélée négativement à la présence d'idées suicidaires et à la réalisation d'au moins une tentative de suicide (cf. tableau 4). Plus les réactions paternelles sont perçues comme négatives, plus les lesbiennes et gais auront de risque de présenter des idéations suicidaires, voire de réaliser une tentative de suicide. À l'inverse, la perception de l'attitude maternelle (facteur 1) n'est liée à aucune des deux variables.

Nous ne trouvons pas de lien entre l'attitude paternelle perçue et les autres variables (gêne en interaction, performance sociale et inquiétudes). A contrario, la perception de la réaction maternelle est corrélée au niveau de gêne en interaction ainsi qu'au score d'inquiétude. Ainsi, moins une jeune ou un jeune se sentira accepté par sa mère, plus elle ou il aura tendance à se sentir gêné lors de ses interactions sociales et préoccupé dans sa vie quotidienne.

\section{Tableau 4}

Corrélations de Bravais Pearson entre l'attitude paternelle, l'attitude maternelle et les différentes variables $(n=82)$

\begin{tabular}{lcc}
\hline & $\begin{array}{c}\text { Facteur 2: } \\
\text { Attitude paternelle }\end{array}$ & $\begin{array}{c}\text { Facteur 1: } \\
\text { Attitude maternelle }\end{array}$ \\
\hline Score d'inquiétude & $r=-0,2$ & $\boldsymbol{r}=\mathbf{- 0 , 4}$ \\
& $p=\mathrm{NS}$ & $\boldsymbol{p} \leq \mathbf{0 , 0 0 0 1}$ \\
Score de gêne & $r=-0,1$ & $\boldsymbol{r}=\mathbf{- 0 , 3}$ \\
& $p=\mathrm{NS}$ & $\boldsymbol{p} \leq \mathbf{0 , 0 1}$ \\
Score de performance & $r=-0,1$ & $r=-0,1$ \\
& $p=\mathrm{NS}$ & $p=\mathrm{NS}$ \\
Présence d'idées suicidaires & $\boldsymbol{r}=\mathbf{- \mathbf { 0 , 3 }}$ & $r=-0,2$ \\
& $\boldsymbol{p} \leq \mathbf{0 , 0 1}$ & $p=\mathrm{NS}$ \\
Réalisation d'au moins une tentative & $\boldsymbol{r}=\mathbf{- 0 , 3}$ & $r=-0,09$ \\
de suicide & $\boldsymbol{p} \leq \mathbf{0 , 0 5}$ & $p=\mathrm{NS}$ \\
\hline
\end{tabular}

\section{DISCUSSION}

En moyenne, les attitudes parentales perçues lors du coming out sont jugées « moyennes » par les participantes et participants. Cependant, au fil du temps, les jeunes de notre échantillon perçoivent une amélioration dans l'attitude de leurs parents, encore plus marquée chez les mères. Lors de l'annonce, les jeunes lesbiennes et gais perçoivent une attitude statistiquement équivalente chez leurs deux parents, mais avec le temps, l'attitude de la mère est vécue comme étant plus rapidement favorable. À la suite du coming out de leur enfant, les parents des gais et lesbiennes pourraient passer par les stades d'adaptation typiques du processus de deuil (Robinson, Walters et Skeen, 1989), se confrontant aux étapes postulées par KüblerRoss (1975) lors de la mort d'un proche, à savoir : le choc, le déni, la culpabilité, la colère et l'acceptation. 
Ils doivent faire le deuil de leur enfant idéal afin d'accéder à l'enfant réel. Ce processus semble se réaliser plus rapidement chez les mères que chez les pères.

L'annonce à la mère est en général réalisée avant le coming out auprès du père. Selon Savin-Williams (2001), la première raison qui pousse les jeunes lesbiennes et gais à ne pas annoncer leur homosexualité à leur père est la distance émotive avec celui-ci, contrairement à la relation avec la mère qui est jugée plus proche. Nos résultats démontrent que la réaction maternelle est perçue comme plus positive que la réaction paternelle si ce n'est lors du coming out. Nous n'avons pas trouvé de lien entre le sexe de l'enfant et la perception de l'attitude des parents, en accord avec l'étude de D'Augelli, Grossman et Starks (2005). Mais ces résultats vont à l'encontre des conclusions de Pilkington et D'Augelli (1995) pour qui les pères réagiraient plus négativement à un fils gai qu'à une fille lesbienne, et les mères auraient des attitudes plus négatives à l'égard de l'homosexualité de leur fille que de leur fils. À ce jour, peu d'études empiriques sont parvenues à déterminer des éléments prédictifs de la réaction parentale au coming out. Il y a lieu de penser que des relations parents-enfants conflictuelles durant l'enfance auront un effet négatif soit sur la décision des jeunes de divulguer leur homosexualité à leurs parents, soit sur l'adaptation des familles chez ceux qui auront décidé de l'annoncer (Julien, 1999).

Nous avons mis en évidence chez les jeunes gais et lesbiennes de notre échantillon des scores moyens d'inquiétude et de gêne lors de leurs interactions sociales qui sont supérieurs aux résultats observés par les auteurs lors de la validation des questionnaires auprès d'individus contrôles. Ces données vont dans le sens des conclusions de Mays et Cochran (2001), qui affirment que les gais et lesbiennes présenteraient une peur prononcée du rejet et auraient tendance à mettre à distance les autres. Les niveaux d'inquiétude et de gêne de nos participantes et participants peuvent être mis en lien avec l'attitude maternelle perçue. Plus la réaction de la mère est perçue comme étant « négative », plus les jeunes sont gênés dans leurs interactions et présentent un niveau d'inquiétude élevé.

La souffrance présente chez les gais et les lesbiennes peut déboucher sur des idéations ou des tentatives de suicide. Selon D'Augelli, Hershberger et Pilkington (2001), $35 \%$ des jeunes lesbiennes, gais, bisexuelles et bisexuels (14-21 ans) ont déjà tenté de se suicider. Au sein de notre échantillon, $31 \%$ des participantes et participants déclarent avoir déjà réalisé au moins une tentative de suicide et $62 \%$ affirment avoir déjà eu des idées suicidaires. Ces pourcentages illustrent l'importance des idéations et des passages à l'acte suicidaires chez les jeunes gais et lesbiennes. Ces résutats vont dans le même sens que le dossier de l'Institut national de prévention et d'éducation pour la santé (Beck, Firdion, Legleye et Schiltz, 2010) et les recherches empiriques (Verdier et Firdion, 2003) menées en France à ce sujet. Nos résultats démontrent qu'une attitude paternelle perçue comme étant mauvaise entretient des liens avec le souhait de mettre fin à ses jours. Plus l'attitude du père sera vécue comme négative, plus le jeune aura des risques de présenter des idéations suicidaires et de réaliser une tentative de suicide.

A contrario, l'acceptation et les liens familiaux forts rendent moins vulnérables les jeunes lesbiennes et gais, qui auraient deux fois moins d'idées suicidaires que ceux qui sont rejetés (Eisenberg et Resnick, 2006). Ryan, Huebner, Diaz et Sanchez (2009) tirent les mêmes conclusions : les gais et lesbiennes rejetées ont plus d'idées suicidaires et plus de conduites sexuelles à risque que ceux et celles qui sont acceptées. Mais ces conclusions méritent d'être précisées, car bien que la perception de l'attitude paternelle soit liée aux passages à l'acte et aux idéations suicidaires, ce n'est pas le cas de l'attitude maternelle. Il est donc 
indispensable de différencier la perception des attitudes maternelles et paternelles, celles-ci n'étant pas corrélées aux mêmes variables.

\section{Limites de l'étude}

Cette étude est exploratoire et présente un certain nombre de limites. Certains outils utilisés dans cette recherche pourraient être affinés et la constitution d'un échantillon plus conséquent, avec un groupe de participantes et participants hétérosexuels, permettrait d'augmenter la validité des résultats obtenus. Le Questionnaire d'attitudes parentales perçues a été constitué dans le cadre de cette étude en absence d'outil, à notre connaissance, évaluant cette dimension. Cet outil en fabrication mériterait d'être amélioré. Il serait intéressant de compléter ces données par l'évaluation des parents, et de questionner les éventuels écarts existants entre la perception des parents et celle de leurs enfants.

\section{CONCLUSION}

Ces résultats mettent l'accent sur le lien existant entre le rejet familial et le mal-être observé chez les jeunes lesbiennes et gais. Des relations familiales conflictuelles et/ou l'appartenance à une minorité sexuelle impliquent un certain nombre de difficultés identitaires. Les dimensions impliquées dans la détresse des jeunes gais et lesbiennes méritent donc encore d'être approfondies, tout comme l'impact des attitudes paternelles et maternelles à l'égard de l'orientation sexuelle de leur enfant. Ces données démontrent la nécessité de créer des dispositifs d'écoute et de médiation familiale afin d'accompagner les jeunes lesbiennes et gais, notamment dans les difficultés qu'ils et elles rencontrent au sein de leur famille. De tels dispositifs sont inexistants en France et peu de professionnel-les sont formé-es aux problématiques particulières des minorités sexuelles. À notre connaissance, seule l'association Le Refuge se propose d'accueillir des jeunes lesbiennes, gais, bisexuelles ou bisexuels en rupture familiale, leur proposant un accompagnement individuel et des médiations familiales. Cette association se développe en France, se déclinant dans différentes villes, mais ne parvient pas à répondre à toutes les demandes. 


\section{RÉFÉRENCES}

Adam, P. (2001). Dépression, tentatives de suicide et prise de risque parmi les lecteurs de la presse gay française. Dans Observatoire du Sida et des Sexualités, Facultés universitaires Saint-Louis (dir.), Vulnérabilité des jeunes gays et lesbiennes et risque de suicide : état de la question et pistes de prévention. Synthèse de la journée d'étude organisée le 17 juillet 2001 (p. 9-21). Bruxelles, Belgique : Observatoire du Sida et des Sexualités, Facultés universitaires Saint-Louis.

Arrindell, W.A., De Groot, P.M. et Walburg, J.A. (1984). Se Schaal voor interpersoonlijk gedrag (SIG) : Handleiding Deel 1. Lisse, Pays-Bas : Swets \& Zeitlinger.

Arrindell, W.A., Sanderman, R., Van der Molen, H., Van der Ende, J. et Mersch, P.P. (1988). The structure of assertiveness: A confirmatory approach. Behaviour Research and Therapy, 4, 337-339.

Arrindell, W.A. et Van der Ende, J. (1985). Cross sample invariance of the structure of self reported distress and difficulty in assertiveness. Advances in Behaviour Research and Therapy, 7, 205-243.

Arrindell, W.A., Van der Ende, J., Sanderman, R., Oosterhof, L., Stewart, R. et Lingsla, M.M. (1999). Normative studies with the Scale for Interpersonal Behaviour (SIB): I. Nonpsychiatric social skills trainees. Personality and Individual Differences, 27, 417-431.

Aseltine, R.H., Gore, S. et Colten, M.E. (1998). The co-occurrence of depression and substance abuse in late adolescence. Development and Psychopathology, 10, 549-570.

Bagley, C. et Tremblay, P. (1997). Suicidal behaviors in homosexual and bisexual males. Crisis, 18, 24-34.

Beck, F., Firdion, J.M., Legleye, S. et Schiltz, M.A. (2010). Les minorités sexuelles face au risque suicidaire : acquis des sciences sociales et perspectives. Saint-Denis, France : Institut national de prévention et d'éducation pour la santé.

Bourdet-Loubère, S. et Pugnière, J.M. (2011). Attirance sexuelle, suicidalité et homophobie intériorisée. Dans D. Welzer-Lang et C. Zaouche Gaudron (dir.), Masculinité : état des lieux (p.113-123). Toulouse, France : Érès.

Bouvard, M., Arrindell, W.A., Guerin, J., Bouchard, C., Rion, A.C., Ducottet, E., . . Cottraux, J. (1999). Psychometric appraisal of the Scale for Interpersonal Behavior in France. Behaviour Research and Therapy, 37, 741-762.

Camilleri, C. (1990). Stratégies identitaires. Paris : Presses Universitaires de France.

Chamberland, L., Émond, G., Julien, D., Otis, J. et Ryan, B. (2010). L'impact de l'homophobie et de la violence homophobe sur la persévérance et la réussite scolaires. Rapport de recherche, programme Actions concertées : ministère de l'Éducation, du Loisir et du Sport et Fonds québécois de la recherche sur la société et la culture. Montréal : Université du Québec à Montréal. Récupéré du site du FQRSC : http://www.fqrsc.gouv.qc.ca/upload/ editeur/RF-LineChamberland.pdf

Clermont, M. et Lacouture, Y. (2000). Orientation sexuelle et santé. Dans Institut de la statistique du Québec (dir.), Enquête sociale de la santé 1998 (p. 219-230). Québec : Institut de la statistique du Québec.

Cochran, S.D. (2001). Emerging issues in research on lesbians' and gay men's mental health: Does sexual orientation really matter? American Psychologist, 56, 931-947.

Corliss, H., Cochran, S. et Mays, V. (2002). Reports of parental maltreatment during childhood in a United States population-based survey of homosexual, bisexual, and heterosexual adults. Child Abuse \& Neglect, 26, 1165-1178.

D'Augelli, A.R., Grossman, A.H. et Starks, M.T. (2005). Parents' awareness of lesbian, gay, and bisexual youths' sexual orientation. Journal of Marriage and Family, 67, 474-482

D’Augelli, A.R., Hershberger, S.L. et Pilkington, N.W. (1998). Lesbian, gay, and bisexual youth and their families: Disclosure of sexual orientation and its consequences. American Journal of Orthopsychiatry, 68, 361-371.

D'Augelli, A.R., Hershberger, S.L. et Pilkington, N.W. (2001). Suicidality patterns and sexual orientation related factors among lesbian, gay, and bisexual youths. Suicide and Life-Threatening Behavior, 31, 250-264.

Davey, G.C.L. (1993). A comparison of three worry questionnaires. Behaviour Research and Therapy, 31, 51-56.

Dugas, M.J., Letarte, H., Rhéaume, J., Freeston, M.H. et Ladouceur, R. (1995). Worry and problem solving: Evidence of a specific relationship. Cognitive Therapy and Research, 19, 109-120.

DuRant, R.H., Krowchuk, D.P. et Sinal, S.H. (1998). Victimization, use of violence, and drug use at school among male adolescents who engage in same-sex sexual behavior. Journal of Pediatrics, 132, 113-118.

Eisenberg, M.E. et Resnick, M.D. (2006). Suicidality among gay, lesbian and bisexual youth: The role of protective factors. Journal of Adolescent Health, 39, 662-668.

Eribon, D. (1999). Réflexions sur la question gay. Paris : Fayard. 
Floyd, F.J., Stein, T.S., Harter, K.S.M., Allison, A. et Nye, C.L. (1999). Gay, lesbian, and bisexual youths: Separationindividuation, parental attitudes, identity consolidation, and well-being. Journal of Youth and Adolescence, 28, 719-739.

Garofalo, R. et Katz, E. (2001). Health care issues of gay and lesbian youth. Current Opinion in Pediatrics, 13, $298-302$.

Garofalo, R., Wolf, R.C., Kessel, S., Palfrey, J. et DuRant, R.H. (1998). The association between health risk behaviors and sexual orientation among a school-based sample of adolescents. Pediatrics, 101, 895-902.

Garofalo, R., Wolf, R.C., Wissow, L.S., Woods, E.R. et Goodman, E. (1999). Sexual orientation and risk of suicide attempts among a representative sample of youth. Archives of Pediatric and Adolescent Medicine, 153, 487-493.

Gibson, P. (1989). Gay male and lesbian youth suicide. Dans M.R. Feinleib (dir.), Report to the Secretary's Task Force on Youth Suicide, vol 3: Prevention and interventions in youth suicide (p.110-142). Rockville, MD : U.S. Department of Health and Human Services.

Hale, W.W., Van der Valk, I., Engles, R. et Meeus, W. (2005). Does perceived parental rejection make adolescents sad and mad? The association of perceived parental rejection with adolescent depression and aggression. Journal of Adolescent Health, 36, 466-474.

Huebner, D.M., Rebchook, G.M. et Kegeles, S.M. (2004). Experiences of harassment, discrimination, and physical violence among young gay and bisexual men. American Journal of Public Health, 94, 1200-1203.

Iwasaki, Y. et Ristock, J. (2007). The nature of stress experienced by lesbians and gay men. Anxiety, Stress and Coping, 20(3), 299-319.

Jouvin, E., Beaulieu-Prévost, D. et Julien, D. (2007). Minorités sexuelles : des populations plus exposées que les autres ? Dans F. Beck, P. Guilbert et A. Gautier (dir.), Baromètre santé 2005 (p. 355-367). Paris, France : Institut national de prévention et d'éducation pour la santé.

Julien, D. (1999). Famille d'origine et homosexualité. Dans M. Simard et J. Alary (dir.), Comprendre la famille : actes du $5^{e}$ symposium québécois de recherche sur la famille (p.210-222). Québec : Presse Universitaire du Québec.

Kessler, R.C., Mickelson, K.D. et Williams, D.R. (1999). The prevalence, distribution, and mental health correlates of perceived discrimination in the United States. Journal of Health and Social Behavior, 40, 208-230.

Kitts, R.L. (2005). Gay adolescents and suicide: Understanding the association. Adolescence, 40, 621-628.

Kübler-Ross, E. (1975). Les derniers instants de la vie. Genève, Suisse : Labor et Fides.

Ladouceur, R., Dugas, M.J., Freeston, M.H., Rhéaume, J., Blais, F., Boisvert, J.M., . . Thibodeau, N. (1999). Specificity of generalized anxiety disorder symptoms and processes. Behavior Therapy, 30(2), 191-207.

Lhomond, B. et Saurel-Cubizolles, M.J. (2003). Orientation sexuelle, violence contre les femmes et santé. Dans C. Broqua, F. Lert, et Y. Souteyrand (dir.), Homosexualités au temps du sida (p.107-130). Paris, France : Agence nationale de recherches sur le sida et les hépatites virales.

Mays, V.M. et Cochran, S.D. (2001). Mental health correlates of perceived discrimination among lesbian, gay, and bisexual adults in the United States. American Journal of Public Health, 91, 1869-1876.

Mellini, L.( 2009). Entre normalisation et hétéronormativité : la construction de l'identité homosexuelle. Déviance et société, 33, 3-26

Ministère français du Travail, de l'Emploi et de la Santé. (2008). Plan « Santé des jeunes ». Dossier de presse. Paris, France : Auteur. Récupéré du site du ministère : http://www.sante.gouv.fr/presentation-du-plan-sante-desjeunes, 474.html

Nungesser, L.G. (1983). Homosexual acts, actors, and identities. New York, NY : Praeger.

Otis, J., Noël, R., Lavoie, R., LeClerc, R., Turmel, B., Alary, M., . . Groupe de recherche Oméga. (2002). Événements suicidaires chez les hommes gais. Vis-à-vie, 10, 15-17.

Pedersen, W. (1994). Parental relations, mental health, and delinquency in adolescents. Adolescence, 29, 975-990.

Piko, B. (2000). Perceived social support from parents and peers: Which is the stronger predictor of adolescent substance use? Substance Use \& Misuse, 35, 617-630.

Pilkington, N.W. et D'Augelli, A.R. (1995). Victimization of lesbian, gay, and bisexual youth in community settings. Journal of Community Psychology, 23, 34-56.

Remafedi, G., Farrow, J.A. et Deisher, R.W. (1991). Risk factors for attempted suicide in gay and bisexual youth. Pediatrics, 87, 869-875.

Robinson, B.E., Walters, L.H. et Skeen, P. (1989). Homosexuality and the family. New York, NY : Harrington Press. 
Rohner, R.P. (2004). The parental "acceptance-rejection syndrome": Universal correlates of perceived rejection. American Psychologist, 59, 830-840.

Rohner, R.P., Khaleque, A. et Cournoyer, D.E. (2003). Cross-national perspectives on parental acceptance-rejection theory. Marriage and Family Review, 35, 85-105.

Russell, S. et Joyner, K. (2001). Adolescent sexual orientation and suicide risk: Evidence from a national study. American Journal of Public Health, 91, 1276-1281.

Ryan, C., Huebner, D., Diaz, R.M. et Sanchez, J. (2009). Family rejection as a predictor of negative health outcomes in white and Latino lesbian, gay, and bisexual young adults. Pediatrics, 123, 346-352.

Saewyc, E.M., Bearinger, L.H., Blum, R.W. et Resnick, M.D. (1999). Sexual intercourse, abuse and pregnancy among adolescent women: Does sexual orientation make a difference? Family Planning Perspectives, 31, 127-131.

Saewyc, E.M., Skay, C.L., Pettingell, S.L., Reis, E.A., Bearinger, L., Resnick, M., . . Combs, L. (2006). Hazards of stigma: The sexual and physical abuse of gay, lesbian, and bisexual adolescents in the United States and Canada. Childhood Welfare Journal, 85, 195-214.

Saewyc, E.M., Poon, C., Wang, N., Homma, Y., Smith, A. et Liebel, A. (2007). Not yet equal: The health of lesbian, gay, \& bisexual youth in BC. Vancouver, BC : McCreary Centre Society.

Saewyc, E.M., Homma, Y., Skay, C.L., Bearinger, L.H., Resnick, M.D. et Reis, E. (2009). Protective factors in the lives of bisexual adolescents in North America. American Journal of Public Health, 99, 110-117.

Savin-Williams, R.C. (1989). Coming out to parents and self-esteem among gay and lesbian youths. Journal of Homosexuality, 18, 1-35.

Savin-Williams, R.C. (1998). The disclosure to families of same-sex attractions by lesbian, gay, and bisexual youths. Journal of Research on Adolescence, 8, 49-68.

Savin-Williams, R.C. (2001). Mom dad I'm gay: How families negotiate coming-out. Washington, DC : American Psychological Association Books.

Savin-Williams, R.C. et Esterberg, K.G. (2000). Lesbian, gay, and bisexual families. Dans D.H. Demo, K.R. Allen et M.A. Fine (dir.), Handbook of family diversity (p. 197-215). New York, NY : Oxford University Press.

Schiltz, M.A. (1995). Parcours des jeunes homosexuels dans le contexte du VIH : la conquête de modes de vie. Population, 52, 1485-1538.

Silenzio, V., Peña, J., Duberstein, P., Cerel, J. et Knox, K. (2007). Sexual orientation and risk factors for suicidal ideation and suicide attempts among adolescents and young adults. American Journal of Public Health, 97, 2017-2019.

SOS Homophobie. (2004). Rapport 2004 sur l'homophobie. Paris, France : Auteur.

Suicide Prevention Resource Center. (2008). Suicide risk and prevention for lesbian, gay, bisexual, and transgender youth. Newton, MA : Education Development Center.

Tallis, F., Eysenck, M. et Mathews, A. (1992). A questionnaire of the measurement of nonpathological worry. Personality and Individual Difference, 13, 162-168.

Velter, A. (2007). Rapport enquête presse gay 2004. Saint-Maurice, France : Agence nationale de recherches sur le sida et les hépatites virales / Institut de veille sanitaire.

Verdier, E. et Firdion, J.M. (2003). Homosexualités et suicide : les jeunes face à l'homophobie. Paris, France : H\&O.

Vincke, J., Bolton, R., Mak, R. et Blank, S. (1993). Coming out and AIDS-related high-risk sexual behavior. Archives of Sexual Behavior, 22, 559-586.

Warner, J., McKeown, E., Griffin, M., Johnson, K., Ramsay, A., Cost, C. et King, M. (2004). Rates and predictors of mental illness in gay men, lesbians, and bisexual men and women. British Journal of Psychiatry, 185, 479-485.

Williams, T., Connolly, J., Pepler, D. et Craig, W. (2005). Peer victimization, social support, and psychosocial adjustment of sexual minority adolescents. Journal of Youth and Adolescence, 34, 471-482.

Willoughby, B.L.B., Malik, N.M. et Lindahl, K. (2006). Parental reactions to their sons' sexual orientation disclosures: The roles of family cohesion, adaptability, and parenting style. Psychology of Men and Masculinity, 7, 14-26. 


\section{ANNEXE \\ Questionnaire des attitudes parentales perçues}

Ce questionnaire est composé de dix items ; pour chaque item, cinq modalités de réponses sont proposées :

1. Très mauvaise

2. Mauvaise

3. Moyenne

4. Bonne

5. Très bonne

Item 1 : Quelle a été la réaction de votre père à l'annonce de votre homosexualité ?

Item 2 : Quelle est son attitude à propos de votre homosexualité aujourd'hui ?

Item 3 : Lorsque vous parlez de votre homosexualité avec lui, comment définiriez-vous le climat de ces conversations?

Item 4 : Lorsque vous évoquez votre partenaire avec lui, comment définiriez-vous le climat de ces conversations?

Item 5 : Quelle est l'attitude générale de votre père à l'égard de votre partenaire ?

Item 6 : Quelle a été la réaction de votre mère à l'annonce de votre homosexualité ?

Item 7 : Quelle est son attitude à propos de votre homosexualité aujourd'hui ?

Item 8 : Lorsque vous parlez de votre homosexualité avec elle, comment définiriez-vous le climat de ces conversations?

Item 9 : Lorsque vous évoquez votre partenaire avec elle, comment définiriez-vous le climat de ces conversations?

Item 10 : Quelle est l'attitude générale de votre mère à l'égard de votre partenaire ? 